\title{
Correction to: Augmented Reality, Virtual Reality, and Computer Graphics
}

\author{
Lucio Tommaso De Paolis and Patrick Bourdot
}

\section{Correction to:}

\section{T. De Paolis and P. Bourdot (Eds.):}

Augmented Reality, Virtual Reality, and Computer Graphics, LNCS 10851, https://doi.org/10.1007/978-3-319-95282-6

In the originally published version of chapters 34 and 37 the name of the author Paulina Ayala was incorrectly spelled as "Paulina X. Ayala". It was corrected to "Paulina Ayala".

The updated online version of these chapters can be found at https://doi.org/10.1007/978-3-319-95282-6_34 https://doi.org/10.1007/978-3-319-95282-6_37 